\title{
Non-factor Quasi-free States of the CAR-algebra
}

\author{
J. MANUCEAU ${ }^{\star}$ and A. Verbeure \\ Instituut voor Theoretische Fysica, Universiteit Leuven, Leuven (Belgium)
}

Received April 10, 1970

\begin{abstract}
A necessary and sufficient condition is given in order that a quasi-free state on the Clifford algebra $\overline{\mathscr{A}}(H, s)$ build on a real separable Hilbert space $(H, s)$ be a factor state.
\end{abstract}

\section{Introduction}

Let $(H, s)$ be a real Hilbert space which is separable (i.e. $H$ is a real vector space and $s$ a real scalar product on $H)$. Let $\overline{\mathscr{A}(H, s)}$ be the CARalgebra constructed on $(H, s)$ i.e. it is the $C^{*}$-algebra generated by the elements $B(\psi)$ where $\psi \rightarrow B(\psi)$ is a real linear map of $H$ into $\overline{\mathscr{A}(H, s)}$ satisfying the anticommutation relations

$$
[B(\psi), B(\varphi)]_{+}=2 s(\psi, \varphi) I
$$

for all $\psi$ and $\varphi$ of $H ; I$ is the unit element in $\overline{\mathscr{A}(H, s)}$.

The quasi-free states $\omega_{A}$ on $\overline{\mathscr{A}(H, s)}$ are those states which are completely determined by an operator $A$ on $H$ such that for all $\psi, \varphi \in H$

$$
\begin{gathered}
\omega_{A}(B(\psi) B(\varphi))=s(\psi, \varphi)+i s(A \psi, \varphi), \\
s(A \psi, \varphi)=-s(\psi, A \varphi) \quad \text { or } \quad A^{+}=-A, \\
\|A\| \leqq 1 .
\end{gathered}
$$

For more details see (1).

A state on a $C^{*}$-algebra is called factor state if it induces a factor G.N.S. representation. In this note we prove that $\omega_{A}$ is not a factor state if and only if the dimension of the kernel of $A$ is odd and

$$
\operatorname{Tr}\left[1-\left(A^{*} A\right)^{\frac{1}{2}}\right]<\infty
$$

\section{The Theorem}

Among the set of quasi-free states $\omega_{A}$ we distinguish two cases: let $\mathfrak{M}_{A}$ be the kernel of the operator $A$, then:

1. dimension of $\mathfrak{M}_{A}$ is even or infinite,

2. dimension of $\mathfrak{M}_{A}$ is odd.

* On leave of absence of Université d'Aix-Marseille, Fac. Sc. St. Charles, Marseille $3^{\mathrm{e}}$ (France). 
a) First case: dimension $\mathfrak{M}_{A}$ is even or infinite.

Let $A=U|A|$ be the polar decomposition of $A$ on $(H, s)$, then on $\mathfrak{M}_{A}^{\perp}=H \ominus \mathfrak{M}_{A}: U^{2}=-1, U^{+}=-U$. Since $A$ is a normal operator, $U$ and $|A|$ commute, and since $\operatorname{dim} \mathfrak{M}_{A}$ is even or infinite we can extend $U$ to $H$ such that its extension $J$ satisfies:

$$
J^{+} J=J J^{+}=1 ; \quad J^{+}=-J ; \quad A=J|A| .
$$

The operator $J$ is a complex structure on $(H, s)$ such that $[A, J]_{-}=0$. Hence the quasi-free state $\omega_{A}$ determined by the operator $A$ is $J$-gauge invariant [1]. It has been proved by several authors $[2,3]$ that gauge invariant quasi-free states are factor states. We summarize:

Proposition 1. Every quasi-free state $\omega_{A}$ on the $C A R$-algebra $\overline{\mathscr{A}(H, s)}$ such that $\operatorname{dim} \mathfrak{M}_{A}$ is even or infinite, is a factor state.

b) Second case: $\operatorname{dim} \mathfrak{M}_{A}$ is odd.

From the work on product states (see e.g. [4]) it follows that without loss of generality we can restrict ourselves to the case that $\operatorname{dim} \mathscr{M}_{A}=1$. The operator $A$ leaves invariant $\mathfrak{M}_{A}$ and $\mathfrak{M}_{A}^{\perp}$, therefore [4] $\omega_{A}$ is a product state

$$
\omega_{A}=\omega_{0} \otimes \omega_{C}
$$

where $\omega_{0}=\omega_{A} \mid \mathscr{A}\left(\mathfrak{M}_{A}, s\right)$ (it is the central quasi-free state of $\mathscr{A}\left(\mathfrak{M}_{A}, s\right)$ ) and $\omega_{C}=\omega_{A} \mid \mathscr{A}_{\left(\mathfrak{M}_{A}^{\perp}, s\right)}\left(\right.$ where $\left.C=A \mid \mathfrak{M}_{A}^{\perp}\right)$. Let $\left(\pi_{0}, \Omega_{0}, \mathscr{H}_{0}\right)$ and $\left(\pi_{C}, \Omega_{C}, \mathscr{H}_{C}\right)$ be the G.N.S. representations, cyclic vectors and representation spaces of $\omega_{0}$, respectively $\omega_{C}$. Let $\left(\pi_{A}, \Omega_{A}, \mathscr{H}_{A}\right)$ be the G.N.S. representation, cyclic vector and representation space induced by the state $\omega_{A}$, then one verifies that

$$
\mathscr{H}_{A}=\mathscr{H}_{0} \bar{\otimes} \mathscr{H}_{C}
$$

$(\bar{\otimes}$ is the completed tensor product of Hilbert spaces),

$$
\begin{aligned}
& \Omega_{A}=\Omega_{0} \otimes \Omega_{C},
\end{aligned}
$$

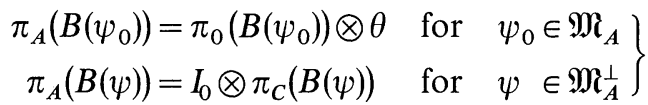

where $I_{0}$ is the unit operator on $\mathscr{H}_{0} ; \theta$ is the unique unitary involutive operator on $\mathscr{H}_{C}$ anticommuting with every element of the form $\pi_{C}(B(\psi))$, $\psi \in \mathfrak{M}_{A}^{\perp}$ such that $\theta \Omega_{C}=\Omega_{C}$ (the existence of the operator $\theta$ is a consequence of the fact that the state $\omega_{C}$ is invariant under the *-automorphism $\gamma$ defined by $\left.\gamma(B(\psi))=-B(\psi), \psi \in \mathfrak{M}_{A}^{\perp}\right)$.

It is proved in (2) and (3. Rideau) that each gauge invariant quasifree state $\omega_{A}$ induces a G.N.S. representation which is quasi-equivalent to the representation induced by a quasi-free state $\omega_{D}$, where $|D|$ has a pure point spectrum. The generalisation of this result to all quasi-free states is immediate. Because two quasi-equivalent representations are 
factor representations if and only if one of them is a factor (5; prop. 5.3.4), from now on we can suppose that $|A|$ has a pure point spectrum. Hence there exists an orthonormal basis $\left\{\psi_{i}\right\}_{i=1,2, \ldots}$ of $\mathfrak{M}_{A}^{\perp}$ such that

then

$$
\text { if } C=J|C|
$$

$$
\begin{gathered}
\psi_{2 i}=J \psi_{2 i-1}, \\
|C| \psi_{2 i-1}=a_{i} \psi_{2 i-1}, \\
|C| \psi_{2 i}=a_{i} \psi_{2 i},
\end{gathered}
$$

and

$$
0<a_{i} \leqq 1 ; \quad i=1,2,3, \ldots .
$$

Using (4) the state $\omega_{C}$ is a product state

$$
\omega_{C}=\bigotimes_{i=1,2, \ldots} \omega_{i}
$$

where $\omega_{i}$ is the restriction of $\omega_{C}$ to $\mathscr{A}\left(H_{i}, s\right) ; H_{i}$ is the subspace generated by $\left\{\psi_{2 i-1}, \psi_{i}\right\}$.

The states $\omega_{i}$ induce a G.N.S. representation determined by the triplet $\left(\pi_{i}, \Omega_{i}, \mathscr{H}_{i}\right)$.

Let

then

$$
\theta_{j}=i \pi_{j}\left(B\left(\psi_{2 j-1}\right) B\left(\psi_{2 j}\right)\right)
$$

$$
\pi_{C}(B(\psi))=\theta_{1} \otimes \theta_{2} \otimes \cdots \otimes \theta_{i-1} \otimes \pi_{i}(B(\psi)) \otimes I_{i+1} \otimes \cdots
$$

for all $\psi \in H_{i} ; I_{j}$ is the unit operator on $\mathscr{H}_{j}$ and

$$
\begin{gathered}
\Omega_{C}=\bigotimes_{i=1}^{\infty} \Omega_{i}, \\
\mathscr{H}_{C}=\bigotimes_{i=1}^{\infty} \mathscr{H}_{i}\left(\text { associated to } \bigotimes_{i=1}^{\infty} \Omega_{i}\right) .
\end{gathered}
$$

Proposition 2. Let $\operatorname{dim} \mathfrak{M}_{A}=1$ and $\operatorname{Tr}(1-|A|)<\infty$, then $\omega_{A}$ is not a factor state.

Proof ${ }^{1}$. It is proved in $[4 ; 2.3 .5]$ that if $\omega_{A}$ is not a factor state, its center is generated by a hermitian, odd element $Z$, such that $Z^{2}=1$. Now we construct explicitly this element.

The operator $|A|$ has a pure point spectrum, hence we can use the infinite product form of the representation induced by $\omega_{C}$ given by (7), (8), and (9). Define the operator

$$
\zeta=\bigotimes_{i=1}^{\infty} \pi_{i}\left(B\left(\psi_{2 i-1}\right) B\left(\psi_{2 i}\right)\right)
$$

\footnotetext{
${ }^{1}$ For a shorter proof, see appendix.
} 
on $\mathscr{H}_{C}$. Now

$$
\begin{aligned}
\sum_{i=1}^{\infty}\left(1-\mid\left(\Omega_{i},\right.\right. & \left.\left.\pi_{i}\left(B\left(\psi_{2 i-1}\right) B\left(\psi_{2 i}\right)\right) \Omega_{i}\right) \mid\right) \\
& =\sum_{i=1}^{\infty}\left(1-a_{i}\right) \\
& =\operatorname{Tr}(I-|C|) .
\end{aligned}
$$

Because $\operatorname{Tr}(I-|A|)<\infty$, we have that $\operatorname{Tr}(I-|C|)<\infty$. Hence the operator $\zeta$ is a bounded operator on $\mathscr{H}_{C}[7]$.

We prove further that $\left.\zeta \in \pi_{C}\left(\mathscr{A}^{(\mathfrak{M}} \mathfrak{M}_{A}^{\perp}, s\right)\right)^{\prime \prime}$. We prove that $\zeta$ is the weak limit of the sequence $\left\{\zeta_{n}\right\}_{n=1,2, \ldots}$

$$
\zeta_{n}=\pi_{C}\left(\prod_{i=1}^{n} B\left(\psi_{2 i-1}\right) B\left(\psi_{2 i}\right)\right) .
$$

The sequence $\left\{\zeta_{n}\right\}_{n=1,2 \ldots}$ is a uniformly bounded sequence, hence it is sufficient to prove the convergence on the set of vectors

$$
\left\{\Omega_{C}, \prod_{j=1}^{k} \pi_{C}\left(B\left(\psi_{i_{j}}\right)\right) \Omega_{C} \mid i_{j} \in \mathbb{N} \quad \text { and } \quad k \in \mathbb{N}\right\} \text {. }
$$

Let

$$
\Psi_{\left(1, \ldots, i_{l}\right)}=\prod_{j=1}^{l} \pi_{C}\left(B\left(\psi_{i_{j}}\right)\right) \Omega_{C}
$$

then we have to prove that

$$
\left|\left(\Psi_{\left(i_{1}, \ldots, i_{l}\right)} ;\left(\zeta-\zeta_{n}\right) \Psi_{\left(r_{1} \ldots r_{k}\right)}\right)\right| \rightarrow 0
$$

as $n$ tends to infinity, for all finite $\left(i_{1}, \ldots, i_{l}\right)$ and $\left(r_{1} \ldots r_{k}\right)$. We take $n>i_{l}$ and $r_{k}$.

Because

$$
\prod_{j=1}^{l} \pi_{C}\left(B\left(\psi_{i_{J}}\right)\right)
$$

commutes or anticommutes with both $\zeta$ and $\zeta_{n}$ depending on whether $l$ is even or odd, (10) is equivalent with

$$
\left|\left(\Omega_{C},\left(\zeta-\zeta_{n}\right) \Psi_{\left(r_{1} \ldots r_{k}\right)}\right)\right| \rightarrow 0 \quad \text { as } n \rightarrow \infty .
$$

The state $\omega_{C}$ is even, hence if $k$ is odd, (11) is trivially satisfied, otherwise put $k=2 m$. It is easily verified that the only non trivial case happens when

$$
\Psi_{\left(2 i_{1}-1,2 i_{1}, \ldots\right)}=\prod_{j=1}^{m} \pi_{C}\left(B\left(\psi_{2 i_{j}-1}\right) B\left(\psi_{2 i_{j}}\right)\right) \Omega_{C} .
$$


In this case

$$
\begin{aligned}
\left|\left(\Omega_{C},\left(\zeta-\zeta_{n}\right) \cdot \Psi_{\left(2 i_{1}-1,2 i_{1}, \ldots\right)}\right)\right| & \left|\prod_{i=1}^{\infty} a_{i}-\prod_{i=1}^{n} a_{i}\right|\left(\prod_{j=1}^{m} a_{i_{j}}\right)^{-1} \\
= & \left(\prod_{i=1}^{n} a_{i}\right)\left(1-\prod_{j=n+1}^{\infty} a_{j}\right)\left(\prod_{j=1}^{m} a_{i_{j}}\right)^{-1} \\
& \leqq 1-\prod_{j=n+1}^{\infty} a_{j}
\end{aligned}
$$

which vanishes when $n$ tends to infinity [7].

Hence

$$
\zeta \in \pi_{C} \overline{\left(\mathscr{A}\left(\mathfrak{M}_{A}^{\perp}, s\right)\right)^{\prime \prime}} .
$$

Now we have two operators $\zeta$ and $\theta$ both anticommuting with the generators

$$
\pi_{C}(B(\psi)), \quad \psi \in \mathfrak{M}_{A}^{\perp}
$$

of

$$
\left.\pi_{C}\left(\overline{\mathscr{A}\left(\mathfrak{M} \mathfrak{T}_{A}^{\perp}, s\right.}\right)\right)^{\prime \prime}
$$

and $\left.I_{0} \otimes \zeta \in \pi_{A}(\overline{\mathscr{A}, H, s})\right)^{\prime \prime}$. One easily verifies that

$$
Z=\pi_{A}\left(B\left(\psi_{0}\right)\right)\left(I_{0} \otimes \zeta\right)=\pi_{0}\left(B\left(\psi_{0}\right) \otimes \theta \zeta \in \pi_{A}(\overline{\mathscr{A}(H, s})^{\prime \prime} \cap \pi_{A}(\overline{\mathscr{A}(H, s})\right)^{\prime} .
$$

Hence $\omega_{A}$ is not a factor state. Q.E.D.

Proposition 3. Suppose that $\operatorname{dim} \mathfrak{M}_{A}=1$ and that $1-|A|$ is not a trace class operator, then the quasi-free state $\omega_{A}$ is a factor state.

Proof. Let $E_{2 n}$ be the subspace of $H$ generated by $\left\{\psi_{0}, \psi_{1}, \ldots, \psi_{2 n-1}\right\}$. The algebra $\overline{\mathscr{A}}(H, s)$ is a UHF algebra and

$$
\left\{\mathscr{M}_{n}=\mathscr{A}\left(E_{2 n} s\right)\right\}_{n}
$$

is an incrasing sequence of $\left(2^{n} \times 2^{n}\right)$-matrix algebras which generates $\mathscr{A}$. A state $\omega$ on $\mathscr{A}$ induces a factor representation of $\mathscr{A}$ if and only if for each $x \in \mathscr{A}$ there is an integer $n>0$ depending only on $x$ such that

$$
|\omega(x y)-\omega(x) \omega(y)| \leqq\|y\|
$$

for all $y \in \mathscr{M}_{n}^{C}[6]\left(\mathscr{M}_{n}^{C}\right.$ consists of all elements of $\mathscr{A}$ commuting with $\left.\mathscr{M}_{n}\right)$.

Let

$$
x=x_{e}+x_{0}
$$

be an arbitrary element of $\mathscr{A}$ such that

$$
\begin{aligned}
& x_{e}=\frac{1}{2}(\gamma(x)+x), \\
& x_{0}=\frac{1}{2}(x-\gamma(x)) .
\end{aligned}
$$


We prove that there exists an integer $n$ such that for all $y \in \mathscr{M}_{n}^{C}$ :

$$
\left|\omega_{A}\left(x_{e} y\right)-\omega_{A}\left(x_{e}\right) \omega_{A}(y)\right| \leqq \frac{1}{2}\|y\|
$$

and

$$
\left|\omega_{A}\left(x_{0} y\right)-\omega_{A}\left(x_{0}\right) \omega_{A}(y)\right| \leqq \frac{1}{2}\|y\| .
$$

1) Because the set $\left\{B\left(\psi_{i}\right) \mid i \in \mathbb{N}=\right.$ the non-negative integers $\}$ generates the algebra $\overline{\mathscr{A}(H, s)}$, there exists an integer $p_{e}$ such that

$$
\begin{gathered}
x_{e}=s_{1}+s_{2}, \\
\left\|s_{2}\right\| \leqq \frac{1}{4},
\end{gathered}
$$

and

$$
s_{1} \in \mathscr{A}_{e}\left(E_{p_{e}}, s\right) .
$$

We note by $\mathscr{A}_{e}$ and $\mathscr{A}_{0}$, the even, respectively the odd part of $\mathscr{A}$. Further for all $q \geqq p_{e}$ and all

$$
y \in \mathscr{A}\left(E_{q}, s\right)^{C}=\overline{\mathscr{A}_{e}\left(E_{q}^{\perp}, s\right)} \oplus \theta_{q} \overline{\mathscr{A}_{0}\left(E_{q}^{\perp}, s\right)}
$$

([4], a) 2.3.1) where $\theta_{q}=i^{q} B\left(\psi_{0}\right) \ldots B\left(\psi_{2 q-1}\right)$,

$$
y=y_{e}+\theta_{q} y_{0}
$$

and we get

$$
\begin{aligned}
\left|\omega_{A}\left(x_{e} y\right)-\omega_{A}\left(x_{e}\right) \omega_{A}(y)\right| & \leqq\left|\omega_{A}\left(s_{1} y\right)-\omega_{A}\left(s_{1}\right) \omega_{A}(y)\right| \\
& +\left|\omega_{A}\left(s_{2} y\right)-\omega_{A}\left(s_{2}\right) \omega_{A}(y)\right| \\
& \leqq\left|\omega_{A}\left(s_{1} y_{e}\right)-\omega_{A}\left(s_{1}\right) \omega\left(y_{e}\right)\right|+\frac{1}{2}\|y\|=\frac{1}{2}\|y\|
\end{aligned}
$$

because firstly $\omega_{A}$ is an even state implying $\omega_{A}\left(s_{1} \theta_{q} y_{0}\right)=0$ and secondly $\omega_{A}$ is a product state implying $\omega_{A}\left(s_{1} y_{e}\right)=\omega_{A}\left(s_{1}\right) \omega_{A}\left(y_{e}\right)$ (for more details see [4], a) 2.1). This proves (13).

2) By the same remark as in 1), there exists an integer $p_{0} \in \mathbb{N}$ such that

$$
\begin{gathered}
x_{0}=t_{1}+t_{2}, \\
\left\|t_{2}\right\| \leqq \frac{1}{4},
\end{gathered}
$$

and

$$
t_{1} \in \mathscr{A}_{0}\left(E_{p_{0}}, s\right) .
$$

Let $r$ be an integer such that $p_{0}<r$ and

$$
y \in \mathscr{A}\left(E_{r}, s\right)^{C}=\overline{\mathscr{A}_{e}\left(E_{r}^{\perp}, s\right)} \oplus \theta_{r} \overline{\mathscr{A}_{0}\left(E_{r}^{\perp}, s\right)}
$$

then

$$
y=y_{e}+\theta_{r} y_{0} .
$$


Hence

$$
\begin{aligned}
\mid \omega_{A}\left(x_{0} y\right) & \omega_{A}\left(x_{0}\right) \omega_{A}(y) \mid \\
\leqq & \left|\omega_{A}\left(t_{1} y\right)-\omega_{A}\left(t_{1}\right) \omega_{A}(y)\right|+\frac{1}{4}\|y\| \\
= & \left|\omega_{A}\left(t_{1} \theta_{r} y_{0}\right)\right|+\frac{1}{4}\|y\|
\end{aligned}
$$

Now $t_{1} \in \mathscr{A}_{0}\left(E_{p_{0}}, s\right)$ and $\omega_{A}$ being a product state

$$
\left|\omega_{A}\left(t_{1} \theta_{r} y_{0}\right)\right|=K \cdot L_{r} \cdot M
$$

where

$$
\begin{aligned}
& K=\left|\omega_{A}\left(t_{1} B\left(\psi_{0}\right) \ldots B\left(\psi_{2 q}\right)\right)\right|, \\
& L_{r}=\left|\omega_{A}\left(B\left(\psi_{2 q+1}\right) \ldots B\left(\psi_{2 r-2}\right)\right)\right|, \\
& M=\left|\omega_{A}\left(B\left(\psi_{2 r-1}\right) y_{0}\right)\right| .
\end{aligned}
$$

Only the value of $K$ depends on $x$; by explicit computation

$$
L_{r}=\prod_{i=q+1}^{r-1} a_{i}
$$

and by increasing the value of $r$, the value of $L_{r}$ can be made as small as we like (remember that $\operatorname{Tr}(1-|A|)=\infty$ implying $\left.\prod_{i=1}^{\infty} a_{i}=0\right)$. Hence we can take $r$ such that

$$
K L_{r}<\frac{1}{4},
$$

finally $\omega_{A}$ being an even state

$$
\begin{aligned}
M & =\left|\omega_{A}\left(B\left(\psi_{2 r-1}\right) y_{0}\right)\right| \\
& =\left|\omega_{A}\left(B\left(\omega_{0}\right) \ldots B\left(\psi_{2 r-2}\right) y\right)\right| \leqq\|y\| .
\end{aligned}
$$

Hence

$$
\left|\omega_{A}\left(x_{0} y\right)-\omega_{A}\left(x_{0}\right) \omega_{A}(y)\right| \leqq \frac{1}{4}\|y\|+\frac{1}{4}\|y\|=\frac{1}{2}\|y\| .
$$

This proves (14).

By taking

$$
n \geqq \max \left(p_{e}, r\right)
$$

$p_{e}$ as defined in 1) and $r$ as in 2) it is satisfied to (12). Q.E.D.

As an immediate consequence of Propositions 1, 2, and 3 we may now formulate the main result.

Theorem. In order that a quasi-free state $\omega_{A}$ on $\overline{\mathscr{A}(H, s)}$ is not a factor state, it is necessary and sufficient that the operator $A$ en $H$ satisfies

(i) $\operatorname{dim}(\operatorname{kernel} A)$ is odd,

(ii) $\operatorname{Tr}\left(I-\left(A^{+} A\right)^{\frac{1}{2}}\right)<\infty$. 


\section{Appendix}

D. Testard informed us about a simpler proof of proposition 2 . With the same notations as above it goes as follows; the state $\omega=\omega_{0} \otimes \omega_{J}$ is not a factor state because the operator $\theta$ belongs to the von Neumann algebra $\pi_{J}\left(\mathscr{A}\left(\mathfrak{M}_{A}^{\perp}, s\right)\right)^{\prime \prime}\left(\pi_{J}\right.$ being irreducible), hence

$$
Z=\pi_{\omega}\left(B\left(\psi_{0}\right)\right) \cdot(I \otimes \theta)=\pi_{0}\left(B\left(\psi_{0}\right)\right) \otimes I
$$

belongs to the center of $\pi_{\omega}$.

Because $\operatorname{Tr}(I-|C|)<\infty$ we have that $\omega_{C}$ is quasi-equivalent to $\omega_{J}[8]$, hence $\omega_{A}$ is quasi-equivalent to $\omega$, implying that $\omega_{A}$ is not factorial.

We reproduced this proof because of its elegance. However we prefer to keep the proof given above, because it contains the explicit construction of the generator of the center.

Acknowledgements. The authors thank M. Sirugue, D. Testard and M. Winnink for fruitful discussions; one of us (A.V.) thanks Professor N. M. Hugenholtz for his kind hospitality at Groningen University (the Netherlands) where this work began and the other (J.M.) Professor F. Cerulus of the Instituut voor Theoretische Natuurkunde, Leuven University (Belgium) where this work was completed.

\section{References}

1. Balslev, E., Manuceau, J., Verbeure, A.: Commun. Math. Phys. 8, 315 (1968).

2. Powers, R. T.: Størmer, E.: Commun. Math. Phys. 16, 1 (1970).

3. Rideau, G.: Commun. Math. Phys. 9, 229 (1968). Sirugue, M., Winnink, M.: Constraints imposed upon a state of a system that satisfies the K.M.S. boundary condition; preprint Marseille.

4. a) Manuceau,J., Rocca, F., Testard, D.: Commun. Math. Phys. 12, 43 (1969).

b) Testard, D.: Type des représentations quasi-libres de l'algèbre de Clifford, preprint.

5. Dixmier, J.: Le $C^{*}$-algèbres et leurs représentations; Paris: Gauthier-Villars 1964.

6. Powers, R. T.: UHF algebras and their applications to the anticommutation relations (Cargèse lecture notes 1969).

7. Neumann, J. von: Comp. Math. 6, 1 (1938).

8. Verbeure, A.: Normal and locally normal quasi-free states (Cargèse Lecture notes 1969).

A. Verbeure

Instituut voor Theoretische Fysica

Departement Natuurkunde

Celestijnenlaan, $200 \mathrm{D}$

3030 Heverlee, Belgium 